Artículo Original

\title{
Evaluación del comportamiento de los profesionales de la salud y su injerencia en los altos niveles de riesgo de biocontaminación en el área de medicina del hospital público Goyeneche Nivel III - Arequipa
}

\author{
JORGE JUVENAL BRAVO HUALLA§ \\ Recibido: 11 junio de 2018 / Aceptado: 09 julio de 2018 \\ $\S E P$. Ingeniería Ambiental, Facultad de Ingeniería y Arquitectura, Universidad Peruana Unión, Perú.
}

\begin{abstract}
Resumen
En la presente investigación se evaluó el comportamiento de los profesionales de la salud en el hospital Goyeneche, área medicina, mediante la Norma Técnica de Salud 096 MINSA/DIGESA V 0.1, con el fin de demostrar la relación entre el comportamiento procedimental en el área de medicina y los niveles de riesgo de biocontaminacion. La problemática es que en el hospital no se cumple con los procedimientos de la Norma Técnica de Salud 096 MINSA/DIGESA V 0.1 y el Art. $N^{\circ} 26$ de la ley $N^{\circ} 29783$, mucho menos se cuida la salud de los profesionales; por lo que se denota altos niveles de biocontaminación. Para la metodología utilizada se siguieron los siguientes pasos: 1) Presentación y aprobación del proyecto en la Universidad Nacional de San Agustín de Arequipa; 2) Presentación y aprobación del proyecto en el hospital Goyeneche III de Arequipa; 3) Evaluación del comportamiento de los profesionales en base a la Norma Técnica de Salud 096 MINSA/DIGESA V 0.1; 4) Criterios para encontrar el Diagnostico de la NTS en cada etapa; 5) Encuesta para revalidar el Diagnostico; 6) Hallazgo de peligros en el área de medicina (varones y mujeres); 7) Hallazgo de Niveles de Riesgo en el área de medicina (varones y mujeres). Los Niveles de Riesgos de biocontaminación encontrados en el área de medicina, están entre 17 a 36, valores que son críticos e inaceptables, según el DS 005-2012-TR de la ley $\mathrm{N}^{\circ}$ 29783. El 83\% de los profesionales manipulan inadecuadamente los residuos biocontaminados. De la encuesta se encontró que el $86 \%$ no sabe qué hacer en caso de sufrir corte por agujas biocontaminadas. La eficiencia del cumplimiento de la NTS es del 7\%. Obtenidos los resultados, la ley 29783 demanda que el área de medicina del hospital debe paralizar sus actividades hasta eliminar los peligros y riesgos hallados en el diagnóstico de la NTS 096 MINSA/DIGESA V 0.1 .
\end{abstract}

Palabras clave: salud, emergencia, MINSA, seguridad, medicina

\begin{abstract}
In the present investigation, the behavior of health professionals in the Goyeneche hospital, medical area, was evaluated through the Technical Health Standard 096 MINSA / DIGESA V 0.1, in order to demonstrate the relationship between the behavior and the procedure in the area of medicine and biocontamination risk levels. The problem is that the hospital does not comply with the procedures of the Technical Health Standard 096 MINSA / DIGESA V 0.1 and Art. No. 26 of Law No. 29783, much less the health of professionals is taken care of; therefore, high levels of biocontamination are indicated. For the Methodology, the following steps were used: 1) Presentation and approval of the project at the National University of San Agustín de Arequipa; 2) Presentation and approval of the project in the Goyeneche III hospital in Arequipa; 3) Evaluation of the behavior of professionals based on the Technical Health Standard 096 MINSA / DIGESA V 0.1; 4) Criteria to find the diagnosis of the NTS in each stage; 5) Survey to validate the Diagnosis; 6) Finding of dangers in the area of medicine (men and women); 7) Finding of Risk Levels in the area of medicine (men and women). The Levels of Biocontamination Risks found in the area of medicine are between 17 and 36, values that are critical and unacceptable, according to DS 005-2012-TR of Law No. $29783.83 \%$ of professionals manipulate insufficiently biocontaminated waste. From the survey it was found that $86 \%$ do not know what to do in case of being cut by biocontaminated needles. The compliance efficiency of the NTS is $7 \%$. Obtained the results, the law 29783 demands that the area of hospital medicine should paralyze its activities until eliminating the risks and risks found in the diagnosis of the NTS 096 MINSA / DIGESA V 0.1
\end{abstract}

*Correspondencia de autor: km. 19 Carretera Central, Ñaña, Lima. E-mail: juvenalbravo@upeu.edu.pe 
Key words: health, emergency, minsa, safety, medicine

\section{INTRODUCCIÓN}

Los establecimientos de salud generan miles de toneladas anuales de desechos. Éstos poseen una gran complejidad, debido a que comprenden, además de desechos comunes, materiales tóxicos, radiactivos e infecciosos ${ }^{[1]}$.

El hospital constituye un tipo especial de ambiente muy distinto del mundo exterior; al servir como centro para el tratamiento de los enfermos, su medio ambiente puede contener una acumulación de enfermedades diversas. ${ }^{[8]}$. Diagnósticos arrojan un mal manejo de los Desechos Sólidos Hospitalarios por lo cual se promueve una adecuada coordinación para que el personal que gerencia y labora en cada una de las clínica; tenga conocimiento, participación y ponga en práctica las normas establecidas para contribuir a una mejor gestión del manejo de los Desechos Sólidos Hospitalarios, sirviendo además como herramienta para la prevención, vigilancia y control de la contaminación e infección microbiana en usuarios, trabajadores y público en general que frecuentan las clínicas, reduciendo los riesgos asociados a la salud por el manejo inadecuado de los desechos ${ }^{[25]}$.

El manejo inadecuado de estos desechos ha contribuido a la crisis ambiental a nivel mundial [...] Los resultados evidencian mal manejo de los Desechos Sólidos Hospitalarios, ausencia de tratamientos y prácticas efectivas que contribuyan a minimizar la contaminación; promoviendo con el aporte de esta información a generar propuestas para un manejo eficiente de DSH ${ }^{[25]}$.

Se evidenció algunos errores comunes en casi todos los servicios hospitalarios. Entre ellos destaca el mal uso de guantes, pues después de manipular fluidos orgánicos o realizar procedimientos, el personal permanece con ellos más tiempo del debido, no realizando cambio oportuno después de la atención de cada paciente, lo que se suma a una menor frecuencia de lavado de manos ${ }^{[2]}$.

Los líquidos residuales no tratados provenientes de centros de salud presentan un riesgo potencial para la salud y el ambiente debido a que transportan sustancias químicas con efectos tóxicos y genotóxicos. La composición de estos líquidos residuales fluctúa continuamente debido a la gran diversidad de medicamentos, desinfectantes, solventes, reactivos de laboratorio, detergentes y otros compuestos vertidos a los mismos ${ }^{[20]}$. Cabe destacar que el manejo deficiente de los desechos peligrosos de hospitales no sólo puede crear situaciones de riesgo que amenacen la salud de la población hospitalaria (personal y pacientes), sino también puede ser causa de situaciones de deterioro ambiental que trasciendan los límites del recinto hospitalario, generar molestias y pérdida de bienestar a la población aledaña al establecimiento y someter a riesgo la salud de aquellos sectores de la comunidad que, directa o indirectamente, lleguen a verse expuestos al contacto con material infeccioso o contaminado, cuando los desechos son trasladados fuera del hospital para su tratamiento o disposición final.

Los desechos peligrosos generados en instituciones de salud requieren de un manejo especial para evitar la transmisión de enfermedades por esa vía, para lo cual es necesario un orden de procedimientos y medios materiales en cada unidad de salud ${ }^{[23]}$.

El presente estudio de investigación evaluó y analizó el comportamiento procedimental de los profesionales del área de medicina del hospital Goyeneche III, llegando a la 
conclusión que existe una relación directa entre los malos procedimientos de segregación de residuos hospitalarios biocontaminados y los altos niveles de riesgo de contaminación, afectando directamente a los profesionales y pacientes en el hospital e indirectamente a la población de Arequipa por el no control de sus residuos sólidos hospitalarios biocontaminados.

\section{MATERIALES Y MÉTODOS}

La metodología para la evaluación del comportamiento de los profesionales en el área de medicina (varones y mujeres) y su relación con los altos niveles de riesgo de biocontaminación estuvo basado en el "Proceso de Identificación de Peligros y Evaluación de Riesgos", dentro del Sistema de Gestión de Seguridad y Salud Ocupacional; enmarcado dentro del D.S. N 005-2012-TR de la ley $\mathrm{N}^{\circ} 29783$.

Evaluación del comportamiento de los profesionales en base a la Norma Técnica de Salud 096 MINSA/DIGESA V 0.1 (Verificación de acciones en horas de trabajo).

Se efectuaron siguiendo los siguientes procedimientos:

Presentación del investigador en el área de medicina por el director del área, a los doctores y enfermeras del área de medicina.

Habiendo elaborado un formato de evaluación en base a la Norma Técnica de Salud 096 MINSA/DIGESA V 0.1, se procedió a observar: procesos, etapas, actividades y tareas. Se evaluaron las acciones (procedimientos) de los profesionales, en cada etapa que plantea la norma, en el área de medicina sin aviso previo.

Se procedió a observar y evaluar sin aviso previo los ambientes de trabajo del área de medicina: lugar de atención al paciente, lugar de reunión de doctores, lugar de reunión de enfermeras, sala de hospitalización de los enfermos (tuberculosis, sida, neumonía, etc.), servicios higiénicos, área de limpieza, cocina y corredores.

Se observó el movimiento de los residuos sólidos hospitalarios biocontaminados en todas las etapas que contemplan la norma: acondicionamiento, almacenamiento primario, segregación, almacenamiento intermedio, transporte interno, almacenamiento central (no se realiza dentro del hospital), tratamiento (no se realiza dentro del hospital), recolección externa (no se realiza dentro del hospital), disposición final (no se realiza dentro del hospital).

Se procedió a observar y evaluar in situ la logística con lo que trabajan los doctores, enfermeras, técnicos, y encargados de limpieza.

Para realizar las evaluaciones a cada procedimiento, se implementó dentro del formato de la Norma Técnica de Salud 096 MINSA/DIGESA V 0.1, criterios de calificación entre 0 (no existe evidencia alguna sobre el tema), 1 (Pobre, no cumple la mayoría de criterios del procedimiento), 2 (regular, no cumple con algunos criterios de evaluación), 3 (bueno, cumple con las principales criterios de evaluación) y 4 (excelente, cumple con todos los criterios de evaluación). 
Así mismo implementó un cuadro en el formato para realizar las observaciones necesarias después de cada evaluación a los procedimientos. Cada evaluación se evidenció con una imagen fotográfica.

Elaboración del Diagnóstico de la Norma Técnica de Salud 096 MINSA/DIGESA V 0.1, en cada etapa

Considerando la Norma Técnica de Salud 096 MINSA/DIGESA V 0.1 y tomando en cuenta el paso 3 de "evaluación", todos los procedimientos que alcanzaban una puntuación de 4 fueron considerados eficientes, y los que tenían una puntuación entre 0 a 3 fueron considerados ineficientes en el cumplimiento del procedimiento.

Los procedimientos que no cumplían con el estándar de la norma, fueron observados, presentaban peligros y riesgos.

El diagnostico se realiza para cada procedimiento dentro de la respectiva etapa.

Una pregunta que nos podría ayudar, sería ¿El presente procedimiento cumple con el $100 \%$ del estándar de la norma? Si no cumple, entonces es deficiente.

\section{Encuesta para revalidar el Diagnostico}

Se realizó una encuesta con el fin de verificar cómo el desconocimiento de las normas vigentes, en el tema de residuos sólidos biocontaminados, puede influir en el comportamiento de los profesionales y producir altos niveles de riesgo de biocontaminación existentes en el área de medicina (Figura 1).

Las preguntas realizadas fueron $i S a b e$ de qué se trata la NTS ${ }^{\circ}$ 096-MINSA/DIGESA ¿Sabe de qué se trata la RM 768-2010-MINSA? ¿Alguna vez ha sufrido alguna corte punzocortante? ¿Está consciente de los riesgos presentes en el ambiente? ¿Se considera capacitado (a) en el tema de Residuos Sólidos Biocontaminados? ¿Qué función cumple en el área?

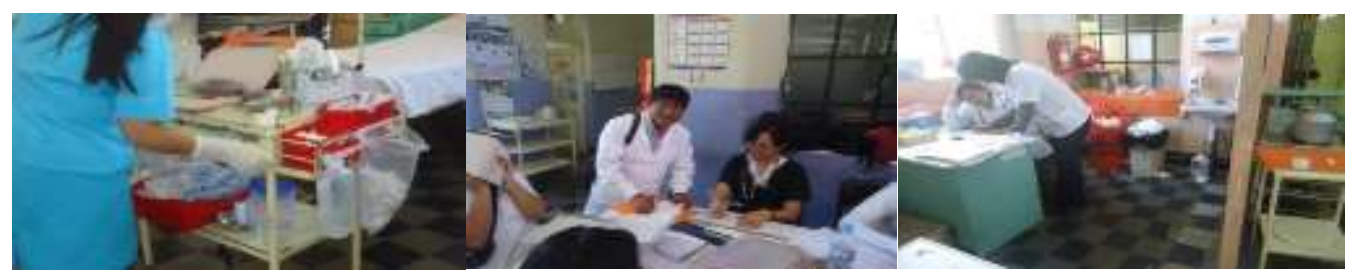

Figura 1. Enfermeras usando plastico como logistica (izquierda), médicos encuestados (centro) y médicos en medio de residuos solidos biocontaminados (derecha)

\section{Hallazgo de peligros en el área de medicina (varones y mujeres)}

Para poder encontrar los riesgos en el área de medicina (varones y mujeres), se tuvo que encontrar los peligros. Para que tenga su aplicación dentro del D.S. $\mathrm{N}^{\circ} 005-2012-\mathrm{TR}$ de la ley $\mathrm{N}^{\circ} 29783$ y encontrar los niveles de riesgo, se consideró el "Proceso de Identificación 
de Peligros y Evaluación de Riesgos", dentro del Sistema de Gestión de Seguridad y Salud Ocupacional. El proceso fue el siguiente: a) Identificar el proceso que se desarrolla; b) Identificar las etapas definidas dentro del proceso; c) Identificar las actividades definidas dentro de las etapas; d) Identificar las tareas definidas dentro de las actividades; e) Identificar los peligros. El resumen del proceso de hallazgo de peligros se observan en la Tabla 1.

Tabla 1. Proceso de Hallazgo de los Peligros

\begin{tabular}{|c|c|c|c|c|c|}
\hline \multicolumn{6}{|c|}{ PROCESO DE HALLAZGO DE LOS PELIGROS } \\
\hline $\mathbf{N}^{\circ}$ & $\begin{array}{l}\text { NOMBRE DEL } \\
\text { PROCESO }\end{array}$ & ETAPA & ACTIVIDADES & TAREAS & PELIGRO \\
\hline 1 & \multirow{9}{*}{$\begin{array}{c}\text { MANEJODE } \\
\text { RESIDUOS SOLIDOS } \\
\text { HOSPITALARIOS } \\
\text { BIOCONTAMINADOS }\end{array}$} & ACONDICIONAMIENTO & ACTIVIDADES DE ETAPA 1 & $\begin{array}{c}\text { TAREAS DENTRO DE LAS } \\
\text { ACTIVIDADES DE LA } \\
\text { ETAPA } 1 \\
\end{array}$ & \begin{tabular}{|c|} 
PELIGROS DE LAS \\
ACTIVIDADES-DE LA ETAPA \\
1 \\
\end{tabular} \\
\hline 2 & & SEGREGACIÓN & ACTIVIDADES DE ETAPA 2 & $\begin{array}{l}\text { TAREAS DENTRO DE LAS } \\
\text { ACTIVIDADES DE LA } \\
\text { ETAPA2 }\end{array}$ & \begin{tabular}{|c|} 
PELIGROS DE LAS \\
ACTIVIDADES-DE LA ETAPA \\
2
\end{tabular} \\
\hline 3 & & ALMACENAMIENTO PRIMARIO & ACTIVIDADES DE ETAPA 3 & $\begin{array}{c}\text { TAREAS DENTRO DE LAS } \\
\text { ACTIVIDADES DE LA } \\
\text { ETAPA3 } 3\end{array}$ & \begin{tabular}{|c} 
PELIGROS DE LAS \\
ACTIVIDADES-DE LA ETAPA \\
3
\end{tabular} \\
\hline 4 & & RECOLECCION Y TRANSPORTE INTERNO & ACTIVIDADES DE ETAPA 4 & $\begin{array}{c}\text { TAREAS DENTRO DE LAS } \\
\text { ACTIVIDADES DE LA } \\
\text { ETAPA4 } 4\end{array}$ & \begin{tabular}{|c|} 
PELIGROS DE LAS \\
ACTIVIDADES-DE LAETAPA \\
4 \\
\end{tabular} \\
\hline 5 & & ALMACENAMIENTO INTERNMEDIO & ACTIVIDADES DE ETAPA 5 & $\begin{array}{c}\text { TAREAS DENTRO DE LAS } \\
\text { ACTIVIDADES DE LA } \\
\text { ETAPA5 } 5\end{array}$ & \begin{tabular}{|c|} 
PELIGROS DE LAS \\
ACTIVIDADES-DE LA ETAPA \\
5
\end{tabular} \\
\hline 6 & & ALMACENAMIENTO FINAL & ACTIVIDADES DE ETAPA 6 & $\begin{array}{l}\text { TAREAS DENTRO DE LAS } \\
\text { ACTIVIDADES DE LA } \\
\text { ETAPA } 6\end{array}$ & $\begin{array}{c}\text { PELIGROS DE LAS } \\
\text { ACTIVIDADES-DE LA ETAPA } \\
6\end{array}$ \\
\hline 7 & & TRATAMIENTO DE RESIDUOS SOLIDOS & ACTIVIDADES DE ETAPA 7 & $\begin{array}{l}\text { TAREAS DENTRO DE LAS } \\
\text { ACTIVIDADES DE LA } \\
\text { ETAPA } 7\end{array}$ & \begin{tabular}{|c|} 
PELIGROS DE LAS \\
ACTIVIDADES-DE LA ETAPA \\
7
\end{tabular} \\
\hline 8 & & RECOLECCION Y TRANSPORTE EXTERNO & ACTIVIDADES DE ETAPA 8 & $\begin{array}{c}\text { TAREAS DENTRO DE LAS } \\
\text { ACTIVIDADES DE LA } \\
\text { ETAPA } 8\end{array}$ & \begin{tabular}{|c} 
PELIGROS DE LAS \\
ACTIVIDADES-DE LA ETAPA \\
8
\end{tabular} \\
\hline 9 & & DISPOSICIÓN FINAL & ACTIVIDADES DE ETAPA 9 & $\begin{array}{l}\text { TAREAS DENTRO DE LAS } \\
\text { ACTIVIDADES DE LA } \\
\text { ETAPA } 9\end{array}$ & $\begin{array}{c}\text { PELIGROS DE LAS } \\
\text { ACTIVIDADES-DE LA ETAPA } \\
9\end{array}$ \\
\hline
\end{tabular}

Hallazgo de Niveles de Riesgo de Biocontaminación en el área de medicina (varones y mujeres) aplicando el D.S. $N^{\circ}$ 005-2012-TR

Identificado los peligros se evaluaron los riesgos existentes. Para hallar los niveles de riesgo de biocontaminación se necesitó evaluarlos en base a los criterios que demanda la norma en el DS 005-2012-TR de la ley $\mathrm{N}^{\circ} 29783$.

A continuación, para hallar los niveles de riesgo de biocontaminación se necesitó los siguientes instrumentos: tabla de Nivel de Probabilidad, tabla de Nivel de Consecuencia Previsible, tabla de Nivel de Exposición al Riesgo, Tabla de Valorización del Riesgo, Matriz de Riesgo (Probabilidad vs Severidad), Tabla resumen de Índices de Estimación del Riesgo y formato del método $\mathrm{N}^{\circ} 2$ de Identificación de Peligros y Evaluación de Riesgos (IPER).

Tabla 2. Nivel de Consecuencia Previsible (Decreto Supremo Nº 005-2012-TR)

\begin{tabular}{ll}
\hline Nivel & Característica \\
\hline Baja & El daño ocurrirá raras veces. \\
Media & El daño ocurrirá en algunas ocasiones. \\
Alta & El daño ocurrirá siempre o casi siempre. \\
\hline
\end{tabular}


Tabla 3. Nivel de Consecuencia Previsible (Decreto Supremo Nº 005-2012-TR)

\begin{tabular}{ll}
\hline Nivel & Características \\
\hline $\begin{array}{l}\text { Ligeramente } \\
\text { dañino }\end{array}$ & $\begin{array}{l}\text { Lesión sin incapacidad: pequeños cortes o magulladuras, irritación de los ojos } \\
\text { por polvo. Molestias e incomodidad: dolor de cabeza, disconfort. }\end{array}$ \\
\hline Dañino & $\begin{array}{l}\text { Lesión con incapacidad temporal: fracturas menores. } \\
\text { Daño a la salud reversible: sordera, dermatitis, asma, trastornos músculo } \\
\text { esqueléticos. }\end{array}$ \\
\hline $\begin{array}{l}\text { Extremadamente } \\
\text { dañino }\end{array}$ & $\begin{array}{l}\text { Lesión con incapacidad permanente: amputaciones, fracturas mayores. Muerte. } \\
\text { Daño a la salud irreversible: intoxicaciones, lesiones múltiples, lesiones } \\
\text { fatales. }\end{array}$ \\
\hline
\end{tabular}

Tabla 4. Nivel de Exposición al Riesgo (Decreto Supremo Nº05-2012-TR)

\begin{tabular}{|c|c|}
\hline Nivel & Característica \\
\hline Esporádicamente 1 & $\begin{array}{l}\text { Alguna vez en su jornada laboral y con periodo corto de tiempo. Al menos } \\
\text { una vez al año. }\end{array}$ \\
\hline Eventualmente & $\begin{array}{l}\text { Varias veces en su jornada laboral aunque sea con tiempos cortos. Al } \\
\text { menos una vez al mes. }\end{array}$ \\
\hline Permanentemente 3 & $\begin{array}{l}\text { Continuamente o varias veces en su jornada laboral con tiempo prolongado. } \\
\text { Al menos una vez al día. }\end{array}$ \\
\hline
\end{tabular}

Tabla 5. Valorización del Riesgo (Decreto Supremo Nº05-2012-TR)

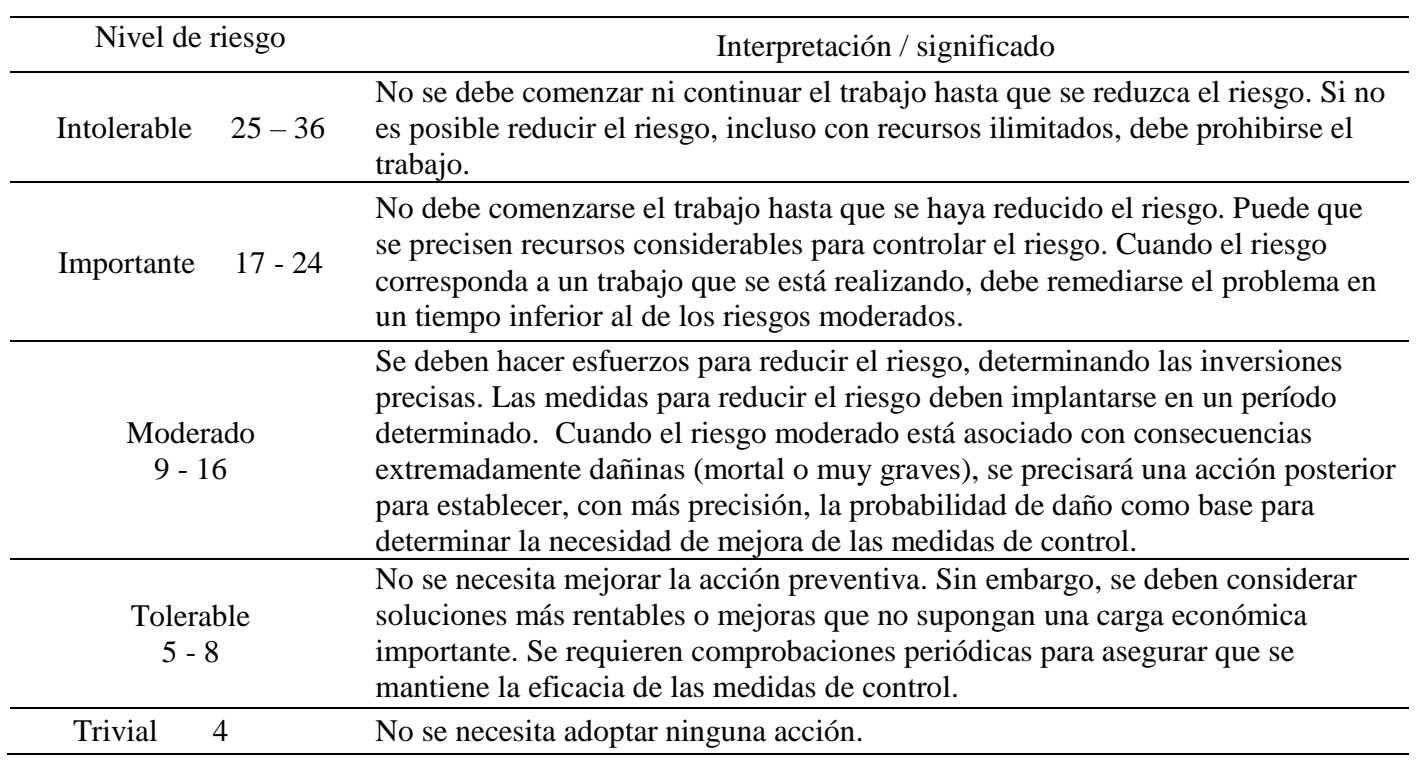


Tabla 6. Matriz de Riesgo (Probabilidad vs Severidad) - Para hallar la Estimación del Nivel de Riesgo de Biocontaminación (Decreto Supremo Nº 005-2012-TR)

\begin{tabular}{cccc} 
& \multicolumn{2}{c}{ CONSECUENCIA } \\
\cline { 2 - 4 } & Ligeramente dañino & Dañino & Extremadamente dañino \\
\cline { 2 - 4 } Baja & Trivial & Tolerable & Moderado \\
\hline \multirow{2}{*}{ Media } & 4 & $5-8$ & $9-16$ \\
\hline \multirow{2}{*}{ Alta } & Tolerable & Moderado & Importante \\
& $5-8$ & $9-16$ & $17-24$ \\
\hline
\end{tabular}

Tabla 7. Cuadro de Estimación del Riesgo (Decreto Supremo Nº 005-2012-TR)

\begin{tabular}{|c|c|c|c|c|c|c|c|}
\hline \multirow{2}{*}{ Índice } & \multicolumn{4}{|c|}{ Probabilidad } & \multirow{2}{*}{$\begin{array}{c}\text { Severidad } \\
\text { (consecuencia) }\end{array}$} & \multicolumn{2}{|c|}{$\begin{array}{l}\text { Estimación del nivel de } \\
\text { riesgo }\end{array}$} \\
\hline & $\begin{array}{l}\text { Personas } \\
\text { expuestas }\end{array}$ & $\begin{array}{l}\text { Procedimientos } \\
\text { existentes }\end{array}$ & Capacitación & $\begin{array}{c}\text { Exposición al } \\
\text { riesgo }\end{array}$ & & $\begin{array}{l}\text { Grado de } \\
\text { riesgo }\end{array}$ & Puntaje \\
\hline \multirow[b]{2}{*}{1} & \multirow[b]{2}{*}{ De 1 A 3} & \multirow[b]{2}{*}{$\begin{array}{l}\text { Existen son } \\
\text { satisfactorios }\end{array}$} & \multirow{2}{*}{$\begin{array}{l}\text { Personal } \\
\text { entrenado } \\
\text { conoce el } \\
\text { peligro y lo } \\
\text { previene }\end{array}$} & $\begin{array}{l}\text { Al menos una vez } \\
\text { al año }(S)\end{array}$ & $\begin{array}{l}\text { Lesión sin } \\
\text { incapacidad (S) }\end{array}$ & Trivial (T) & 4 \\
\hline & & & & $\begin{array}{l}\text { Esporádicamente } \\
\text { (SO) }\end{array}$ & $\begin{array}{l}\text { Disconfort / } \\
\text { Incomodidad } \\
\text { (ch) }\end{array}$ & $\begin{array}{l}\text { Tolerable } \\
\text { (TO) }\end{array}$ & $\begin{array}{l}\text { De } 5 \text { a } \\
8\end{array}$ \\
\hline \multirow{2}{*}{2} & \multirow{2}{*}{ De 4 a 12} & \multirow{2}{*}{$\begin{array}{l}\text { Existen } \\
\text { parcialmente y no } \\
\text { son satisfactorios o } \\
\text { suficientes }\end{array}$} & \multirow{2}{*}{$\begin{array}{l}\text { Personal } \\
\text { parcialmente } \\
\text { entrenado, conoce } \\
\text { el peligro pero no } \\
\text { toma acciones de } \\
\text { control }\end{array}$} & $\begin{array}{l}\text { Al menos una vez al } \\
\text { mes (s) }\end{array}$ & $\begin{array}{l}\text { Lesión con } \\
\text { incapacidad } \\
\text { temporal }(\mathrm{S})\end{array}$ & $\begin{array}{l}\text { Moderado } \\
\text { (M) }\end{array}$ & $\begin{array}{l}\text { De } 9 \text { a } \\
16\end{array}$ \\
\hline & & & & $\begin{array}{l}\text { Eventualmente } \\
\text { (SO) }\end{array}$ & $\begin{array}{l}\text { Daño a la salud } \\
\text { reversible }\end{array}$ & $\begin{array}{l}\text { Importante } \\
\text { (IM) }\end{array}$ & $\begin{array}{l}\text { De } 17 \text { a } \\
24\end{array}$ \\
\hline \multirow[b]{2}{*}{3} & \multirow[b]{2}{*}{ Más de 12} & \multirow[b]{2}{*}{ No existen } & \multirow{2}{*}{$\begin{array}{l}\text { Personal no } \\
\text { entrenado, no } \\
\text { conoce el peligro, no } \\
\text { toma acciones de } \\
\text { control }\end{array}$} & $\begin{array}{l}\text { Al menos una vez al } \\
\text { día }(S)\end{array}$ & $\begin{array}{l}\text { Lesión con } \\
\text { incapacidad } \\
\text { permanente (S) }\end{array}$ & $\begin{array}{l}\text { Intolerable } \\
\text { (IT) }\end{array}$ & $\begin{array}{l}\text { De } 25 \text { a } \\
36\end{array}$ \\
\hline & & & & $\begin{array}{l}\text { Permanentemente } \\
\text { (SO) }\end{array}$ & $\begin{array}{l}\text { Daños a la } \\
\text { salud } \\
\text { Irreversible }\end{array}$ & & \\
\hline
\end{tabular}

Para el hallazgo de los Niveles de Riesgo de Biocontaminación se consideraron: el proceso hallado, las etapas encontradas dentro del proceso, las actividades a evaluar dentro de las etapas, las tareas encontradas dentro de las actividades, los peligros hallados dentro de las tareas, los riesgos analizados por la presencia de los peligros, hallazgo de los Niveles de Riesgo por la presencia de residuos hospitalarios biocontaminados en el proceso. 


\section{RESULTADOS Y DISCUSIÓN}

Tabla 8. Resultados de evaluación de procedimientos en las etapas de manejo de Residuos Sólidos Hospitalarios Biocontaminados de la NTS N 096-MINSA/DIGESA - Hospital Goyeneche - Arequipa

\begin{tabular}{|c|c|c|c|c|c|c|c|c|}
\hline $\begin{array}{l}\text { 芯 } \\
\text { 芒 }\end{array}$ & 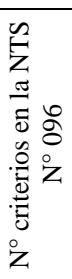 & 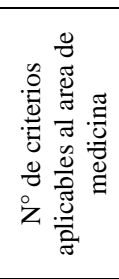 & 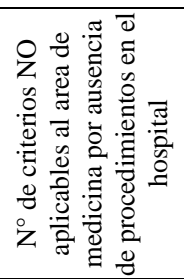 & 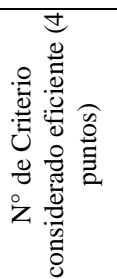 & 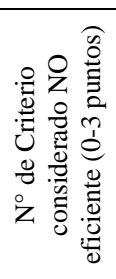 & 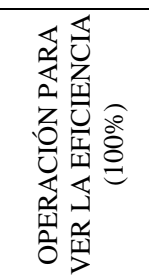 & 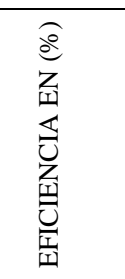 & 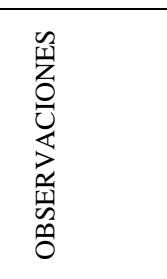 \\
\hline I & 20 & 20 & 0 & 4 & 16 & $4(100) / 20$ & $20 \%$ & \\
\hline II & 10 & 6 & 4 & 0 & 6 & $0(100) / 6$ & $0 \%$ & $\bar{\omega} \stackrel{0}{\tilde{0}} \overline{0}$ \\
\hline III & 7 & 5 & 2 & 0 & 5 & $0(100) / 5$ & $0 \%$ & $\bar{\sigma} \approx \bar{\pi} \overline{0}$ \\
\hline IV & 15 & 12 & 3 & 0 & 12 & $0(100) / 12$ & $0 \%$ & 苂 \\
\hline V & 17 & 17 & 0 & 0 & 17 & $0(100) / 17$ & $0 \%$ & \\
\hline VI & 23 & \multirow{4}{*}{\multicolumn{6}{|c|}{$\begin{array}{l}\text { No Existe ningun tipo de procedimientos en las Etapas VI, VII, VIII, IX, lo cual } \\
\text { es preocupante por la inexistencia de Gestión de Manejo de Residuos Sólidos } \\
\text { Hospitalarios Biocontaminados. }\end{array}$}} & \multirow{4}{*}{ 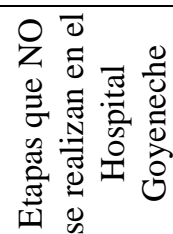 } \\
\hline VII & 7 & & & & & & & \\
\hline VIII & 13 & & & & & & & \\
\hline IX & 8 & & & & & & & \\
\hline
\end{tabular}

No se cumplen con los procedimientos correctos etapa por etapa. Al realizar la evaluación, vemos que la gestión de manejo de residuos sólidos en el hospital Goyeneche es sumamente crítico, donde su EFICIENCIA de manejo de residuos sólidos biocontaminados es del 0\% (cero por ciento) en las etapas II, III, IV y V (Tabla 9). Así mismo se concluye que en la primera etapa solo llega a un 20\%. El hospital Goyeneche a partir de la quinta etapa se desentiende completamente, no sabiendo cómo se tratan los residuos sólidos biocontaminados cuando salen de sus ambientes.

Tabla 9. Eficiencia de las etapas I, II, III, IV y V - Resultados de la evaluación de procedimientos en las etapas de manejode RR.SS hospitalarios biocontaminados de la NTS N $096-$ MINSA/DIGESA aplicados al hospital Goyeneche - Arequipa

\begin{tabular}{cc}
\hline Etapa & Eficiencia \\
\hline I & $20 \%$ \\
II & $0 \%$ \\
III & $0 \%$ \\
IV & $0 \%$ \\
V & $0 \%$ \\
\hline
\end{tabular}

Teniendo en cuenta todo el proceso de manejo de residuos sólidos hospitalarios biocontaminados en el hospital Goyeneche, en sus 5 etapas, tiene una muy preocupante eficiencia del 7\% (Tabla 10). 
Tabla 10. Resultados de encuesta realizada ${ }^{a}$ respecto al manejo de residuos sólidos biocontaminados por el personal en el área de medicina

\begin{tabular}{lcccc}
\hline Pregunta/respuesta & $\mathrm{Si}$ & $\%$ & No & $\%$ \\
\hline ¿Sabe de que se trata la NTS N & 7 & 17 & 35 & 83 \\
¿Sabe de que se trata la RM N ${ }^{\circ}$ 768-MINSA/DIGESA-V.01? & 6 & 14 & 36 & 86 \\
¿Alguna vez a sufrido algún herida punzocortante? & 18 & 43 & 24 & 57 \\
¿Esta conciente de los riesgos presentes en el ambiente? & 41 & 98 & 1 & 2 \\
¿Se considera capacitado (a) en el tema de RRSS Biocontaminados? & 27 & 64 & 15 & 36 \\
\hline a En base a 42 personas encuestadas & & & &
\end{tabular}

${ }^{a}$ En base a 42 personas encuestadas

Tabla 11. Profesionales que trabajan en el área de medicina

\begin{tabular}{lcr}
\hline Profesión & $\mathrm{N}^{\circ}$ & $\%$ \\
\hline Operario de limpieza & 4 & 9.52 \\
Jefatura de Limpieza & 1 & 2.38 \\
Jefa de Oficina de Docencia e Investigación & 1 & 2.38 \\
Médico Jefe de Epidemiología & 1 & 2.38 \\
Estudiante de Medicina & 10 & 23.81 \\
Doctora Asistente - Medicina & 1 & 2.38 \\
Tecnica Administrativa & 1 & 2.38 \\
Interno de Medicina & 7 & 16.67 \\
Tecnica Enfermera & 3 & 7.14 \\
Medico Residente & 2 & 4.76 \\
Rotante de Medicina Legal & 1 & 2.38 \\
Medico & 3 & 7.14 \\
Interna de Enfermeria & 1 & 2.38 \\
Enfermera Asistencial & 3 & 7.14 \\
Enfermera Jefe & 1 & 2.38 \\
Estudiante de farmaceutica & 1 & 2.38 \\
Interna de Nutrición & 1 & 2.38 \\
\hline Total & 42 & 100.00 \\
\hline
\end{tabular}

\section{Interpretación}

El universo de encuestados fueron 42, es decir todos los que realizan alguna labor en el área de medicina varones y mujeres.

Son $83 \%$ de los encuestados que no conocen el proceso de manipulación de los residuos sólidos biocontaminados.

La estadística lanzo que el $86 \%$ de los encuestados no saben qué hacer en caso de sufrir un accidente por corte de objeto punzocortante biocontaminado.

Del $100 \%$ de los encuestados, el $57 \%$ alguna vez ha sufrido alguna herida por objeto biocontaminado.

El 98\% de los encuestados esta consiente de los riesgos en el área, pero por los valores antes obtenidos no saben qué hacer frente a ellos.

Del $100 \%$ de encuestados, el $88 \%$ son personas formadas en el tema de la salud. Este porcentaje es muy alto y a la vez preocupante que los encargados del cuidado de la salud, están provocando riesgos de biocontaminación en el área de medicina en el Hospital Goyeneche III. 
Tabla 11. Resumen de niveles de riesgo de acuerdo a la ley 29783 y el Nº05-2010-TR (Etapa por etapa)

\begin{tabular}{|c|c|c|}
\hline \multicolumn{3}{|c|}{ INDICATIVO DE NIVEL DE RIESGO } \\
\cline { 1 - 2 } PUNTAJE & NIVEL DE RIESGO & RIESGO SIGNIFICATIVO \\
\hline $25-36$ & INTOLERABLE (IT) & \multirow{2}{*}{ SI } \\
\hline $17-24$ & IMPORTANTE (IM) & \multirow{2}{*}{ NO } \\
\hline $09-016$ & MODERADO (M) & \\
\cline { 1 - 2 } $04-008$ & $\begin{array}{c}\text { TRIVIAL (T) - } \\
\text { TOLERABLE (TO) }\end{array}$ & \\
\hline
\end{tabular}

Tabla 12. Evaluación de niveles de riesgo de acuerdo a la ley 29783 y D.S. N005-2010-TR (según etapa)

\begin{tabular}{|c|c|c|c|c|}
\hline Etapa & Puntaje & Nivel de riesgo & $\begin{array}{c}\text { Riesgo } \\
\text { significativo }\end{array}$ & Interpretación \\
\hline \multirow{2}{*}{$\begin{array}{c}\text { I } \\
\text { Adecuación }\end{array}$} & $17-24$ & $\begin{array}{l}\text { Importante } \\
\text { (IM) }\end{array}$ & SI & \multirow{2}{*}{$\begin{array}{c}\text { Según la ley } 29783\left(\mathrm{DS} \mathrm{N}^{\circ} 005-\right. \\
\text { 2010-TR)deben paralizar las } \\
\text { actividades hasta que se } \\
\text { solucionen las observaciones }\end{array}$} \\
\hline & $25-36$ & Intolerable (IT) & SI & \\
\hline $\begin{array}{c}\text { II } \\
\text { Segregación }\end{array}$ & $25-36$ & Intolerable (IT) & SI & $\begin{array}{c}\text { Según la ley } 29783\left(\mathrm{DS} \mathrm{N}^{\circ} 005-\right. \\
\text { 2010-TR)deben paralizar las } \\
\text { actividades hasta que se } \\
\text { solucionen las observaciones }\end{array}$ \\
\hline $\begin{array}{l}\text { III } \\
\text { Recip. primarios }\end{array}$ & $25-36$ & Intolerable (IT) & SI & $\begin{array}{c}\text { Según la ley } 29783\left(\mathrm{DS} \mathrm{N}^{\circ} 005-\right. \\
\text { 2010-TR)deben paralizar las } \\
\text { actividades hasta que se } \\
\text { solucionen las observaciones }\end{array}$ \\
\hline \multirow{2}{*}{$\begin{array}{l}\text { IV } \\
\text { Recolección y } \\
\text { transporte }\end{array}$} & $17-24$ & $\begin{array}{l}\text { Importante } \\
\text { (IM) }\end{array}$ & SI & \multirow{2}{*}{$\begin{array}{c}\text { Según la ley } 29783\left(\mathrm{DS} \mathrm{N}^{\circ} 005-\right. \\
\text { 2010-TR)deben paralizar las } \\
\text { actividades hasta que se } \\
\text { solucionen las observaciones }\end{array}$} \\
\hline & $25-36$ & Intolerable (IT) & SI & \\
\hline \multirow{2}{*}{$\begin{array}{l}\mathrm{V} \\
\text { Almacenamiento } \\
\text { intermedio }\end{array}$} & $17-24$ & $\begin{array}{l}\text { Importante } \\
\text { (IM) }\end{array}$ & SI & \multirow{2}{*}{$\begin{array}{c}\text { Según la ley } 29783\left(\mathrm{DS} \mathrm{N}^{\circ} 005-\right. \\
\text { 2010-TR)deben paralizar las } \\
\text { actividades hasta que se } \\
\text { solucionen las observaciones }\end{array}$} \\
\hline & $25-36$ & Intolerable (IT) & SI & \\
\hline
\end{tabular}

Observamos que en la primera etapa (adecuación), los niveles de riesgo de biocontaminación alcanzados son Importante e Intolerable, ambos niveles de riesgo son muy altos, lo que me lleva a detener cualquier actividad que se está desarrollando. Así lo especifica la Ley 29783. En la segunda etapa (segregación), también debería detenerse toda actividad. En la tercera etapa, también debería detenerse toda actividad ya que los niveles de riesgo son los más elevados. En la cuarta y quinta etapa también debería detenerse cualquier actividad pues tienen niveles de riesgo altísimos. Los resultados obtenidos demuestran que los niveles de riesgo de biocontaminación en el área de medicina del hospital Goyeneche son muy altos, encontrándolos INTOLERABLES E IMPORTANTES. Esto lleva a paralizar cualquier actividad que se esté desarrollando en el área de medicina hasta que se levanten las observaciones que demanda la ley 29783 y el DS N ${ }^{\circ} 005-2010$ TR, esto debido al incumplimiento de la NTS $\mathrm{N}^{\circ}$ 096-MINSA/DIGESA-V.01. Si esto no sucediera el representante del hospital Goyeneche podría ir a la cárcel, esto de acuerdo a Ley 29783. 


\section{CONCLUSIONES}

Después de analizar las acciones de los profesionales de salud, en el área de medicina (hombres y mujeres) del hospital Goyeneche III de Arequipa, y bajo el amparo de ley 29783 (Ley de Seguridad y Salud en el Trabajo) y su reglamento DS 005-2010-TR, se llegó a la conclusión que las malas prácticas en la segregación de residuos hospitalarios provocan altos niveles de riesgo de biocontaminación, por la presencia de agentes patógenos (virus, bacterias). Los niveles de riesgo obtenidos son importantes e intolerables.

Se ha realizado la evaluación de actos y condiciones subestándar, como resultado de esta evaluación se obtuvo la línea base del manejo de residuos sólidos biocontaminados. Los resultados obtenidos fueron CRITICOS E INACEPTABLES. Se realizan malos procedimientos de manejo y recolección de los residuos sólidos biocontaminados. Las condiciones laborales son pésimas. La población arequipeña esta propensa a una contaminación cruzada.

Después de realizado el presente estudio, y entender las causas que propician la presencia de peligros, riesgos y altos niveles de riesgo en el área de medicina, se propone un plan de capacitaciones y entrenamiento con el objetivo de corregir los actos y condiciones subestándar y así disminuir los altos niveles de riesgo. Se busca que la capacitación y entrenamiento sea real y constante. Observando la crisis en el manejo de los residuos sólidos hospitalarios biocontaminados, en el hospital Goyeneche III, proponemos una mejora continua en la gestión del manejo de residuos sólidos biocontaminados. Proponemos implantar el Ciclo PDCA (Planear, Hacer, Verificar, Ajustar), con el fin de elevar los estándares de gestión del manejo de residuos sólidos hospitalarios biocontaminados.

Existe una gran necesidad de implementar dentro del hospital Goyeneche III, una autoclave y un triturador para solucionar la gran amenaza de pandemia a la que está expuesto el hospital y la población arequipeña.

\section{Recomendación}

Habiendo evaluado los parámetros de regulación de Salud y Seguridad Ocupacional, NTS $\mathrm{N}^{\circ}$ 096-MINSA/DIGESA-V.01. ley 29783 y el D.S. $\mathrm{N}^{\circ} 005-2010-\mathrm{TR}$, el hospital Goyeneche del área de medicina, debe suspender sus actividades hasta que cumpla con las normas mínimas establecidas.

\section{Referencias}

1. Cecilia Cifuentes, Silvia Iglesias, Gestión ambiental de residuos sólidos hospitalarios del hospital Cayetano Heredia, 2008

2. Víctor Soto, Enrique Olano, Conocimiento y cumplimiento de medidas de bioseguridad en personal de enfermería. Hospital Nacional Almanzor Aguinaga Chiclayo, 2002

3. Eduardo Jesús Yactayo Infante, Modelo de Gestión Ambiental para el manejo de residuos sólidos hospitalarios, 2013

4. Felicia Nemathagaa, Sally Maringa, Luke Chimukab, Prácticas de gestión de residuos sólidos hospitalarios en la provincia de Lipopo, Sudafrica: Un estudio de casos de hospitales, 2008

5. Revista de Salud Ciencia y Salud, abril 1995. 
6. Cristina de Martos, El Mundo es Salud 2007.

7. Víctor Gómez, Prensa Libre, noviembre 2015.

8. Dr. Franklin H. Top, 1970

9. Ley General de Salud. Ley N ${ }^{\circ}$ 26842. Congreso de la República del Perú, [15-071997].

10. Ley General de Residuos Sólidos. Ley N²7314. Presidencia de la República, [1007-2000].

11. Norma Técnica de Salud. Norma $N^{\circ}$ 096-MINSA/DIGESA-V.01. Resolución Ministerial N $\mathrm{N}^{\circ}$ 554-2012/MINSA, [06-07-2012]. Ley de Seguridad y Salud en el Trabajo. Ley 29783. Presidencia de la república, [19-08-2011].

12. Decreto Supremo 005-2012-TR. Reglamento de la Ley 29783, Ley de Seguridad y Salud en el Trabajo. Ministerio de Trabajo y Promoción del Empleo, [25-04 2012].

13. Plan de Manejo de Residuos Sólidos del Hospital Goyeneche III. Resolución Directoral, 2015.

14. G.D. Cataluña, E. Manual para la Identificación de Riesgos Laborales. Dirección General de Relaciones Laborales de Cataluña. Cataluña, España; 2006.

15. Karina Takahashi. Adecuado manejo y tratamiento de los residuos sólidos biocontaminados. Lima; 2014.

16. Manuel García P, Carlos Quispe A. Mejora Continua de la Calidad en los Procesos, Volumen (6) 1: pp. 89-94.

17. Mogrovejo Gonzales D. Prevención de Riesgos Laborales para el área de Limpieza Pública de la Municipalidad Provincial de Arequipa [tesis]. Arequipa: Servicio de biblioteca, Universidad Tecnológica del Perú - Filial Arequipa; 2015.

18. Juárez Herrera C. Propuesta de mejora del sistema de gestión de la seguridad y salud ocupacional basado en el D.S. 055-2010-EM, para una planta de beneficio de procesamiento de oro. Chala-Arequipa [tesis]. Arequipa: Servicio de biblioteca, Universidad Tecnológica del Perú - Filial Arequipa; 2015.

19. Derecho a la Salud y protección al discapacitado, derecho a la seguridad social, economía social de mercado, protección al consumidor. Artículos 7, 10, 58, 65. Constitución Política del Perú, [29-12-1993].

20. Marta Paz, AnahíMagdaleno, Carina Tornello, Natalia Balbis y Juan Moretton, genotoxicidad y determinación de compuestos tóxicos en un residuo liquido hospitalario de Buenos Aires, Argentina, 2008

21. Luis Carlos Riofrío Cortés, Janneth Torres Agredo, Herramienta para evaluar la gestión de residuos hospitalarios, Colombia, 2016

22. Víctor Soto, Enrique Olano, Conocimiento y cumplimiento de medidas de bioseguridad en personal de enfermería. Hospital Nacional Almanzor Aguinaga. Chiclayo 2002

23. Raquel de los Ángeles Junco Díaz1 e Ing. Doraida S. Rodríguez Sordía, Desechos hospitalarios: Aspectos Metodológicos de su manejo, 2000

24. Edgar Chirinos, Eduarda Rivero, Aurora Goyo, Elita Méndez, Carlos Figueredo, Indicadores de gestión para medir la eficiencia hospitalaria, 2008

25. Nancy Reyes de Borjas y Zuleima Chirinos, Manejo de desechos sólidos hospitalarios en clínicas privadas del municipio Miranda, estado Falcón, Venezuela, 2010

26. García Fenton, Vania; Fleitas Triana, María Sonia; García-Rovés Placer, Bibiana; Pérez Rodríguez, Nancy, Tecnología para la gestión integrada de capital humano hospitalaria, 2009

27. Rodrigo García Alvarado, Felipe Baesler Abufarde, Pedro Rodriguez Moreno, Mauricio Pezo Bravo, Análisis del diseño y gestión de servicios de emergencia hospitalaria en Chile, 2003 